\title{
Patch amperometry: high-resolution measurements of single-vesicle fusion and release
}

\author{
Gregor Dernick 1,3 , Liang-Wei Gong ${ }^{1,3}$, Lucia Tabares', Guillermo Alvarez de Toledo ${ }^{2} \&$ \\ Manfred Lindau ${ }^{1}$
}

${ }^{1}$ School of Applied and Engineering Physics, Cornell University, Ithaca, New York 14850, USA. ${ }^{2}$ Department of Physiology and Biophysics, School of M edicine, University of Seville, E-41009 Seville, Spain. 3Present addresses: F. H offmann-La Roche Ltd., CH - 4070 Basel, Switzerland (G.D.), and Department of Cell Biology and Howard Hughes M edical Institute, Yale University School of M edicine, New Haven, Connecticut 06510, USA (L.-W.G.). Correspondence should be addressed to M .L. (ml95@cornell.edu).

Patch amperometry is a new technique for the observation of single-vesicle exocytosis. Exocytosis of single vesicles as small as $50 \mathrm{~nm}$ in diameter can be detected by cell-attached patch-clamp admittance measurements ${ }^{1-4}$ indicating fusion of vesicles with the plasma membrane or by amperometry with a carbon fiber electrode (CFE) ${ }^{5-9}$ indicating release of oxidizable molecules such as catecholamines. The admittance measurement provides the membrane capacitance that increases in proportion to the membrane area because of the incorporation of the vesicle into the patch membrane. It also reveals the fusion pore conductance during an exocytotic event, giving an estimate of fusion pore dimensions. Amperometry provides the amount and time course of release of molecules that are readily oxidizable such as dopamine, norepinephrine or serotonin. This technique is capable of detecting as little as a few thousand molecules ${ }^{8,9}$. It also resolves the flux of catecholamines through a narrow fusion pore in a so-called foot signal that precedes rapid release indicated by an amperometric spike ${ }^{6}$. Patch amperometry combines high-resolution patch capacitance measurements with amperometry by placing the amperometric detector inside the patch pipet ${ }^{10}$. The method provides precise information on single-vesicle size and quantal content, fusion pore conductance and permeability of the pore for catecholamines ${ }^{10-14}$. Thus, it is a unique tool to investigate the mechanisms that modulate quantal size and the effect of molecular manipulations affecting the properties of the fusion pore. Here we provide step-by-step instructions for the application of this method.

\section{MATERIALS}

\section{REAGENTS}

Cells to bestudied plated onto $12 \mathrm{~mm}$-diameter coverslips (for example, bovinechromaffin cells prepared as described ${ }^{15}$ )

Bath and pipet solutions appropriate for cell type and experimental conditions to be studied. Bath solution (for chromaffin cells): $140 \mathrm{mM} \mathrm{NaCl}, 5 \mathrm{KCl} \mathrm{mM}, 5 \mathrm{mM} \mathrm{CaCl}, 1 \mathrm{mM} \mathrm{M} \mathrm{gCl} 2,10 \mathrm{mM} \mathrm{HEPES}-\mathrm{NaOH}, 10 \mathrm{mM}$ glucose (pH 7.3). Pipet solution (for chromaffin cells): $50 \mathrm{mM} \mathrm{NaCl}, 100 \mathrm{mM}$ tetraethylammonium ion (TEA)-Cl, $5 \mathrm{mM} \mathrm{KCl}, 10 \mathrm{mM} \mathrm{CaCl}, 1 \mathrm{mM} \mathrm{M} \mathrm{gCl}$, 10 mM HEPES-NaOH (pH 7.3)

Sticky wax (Sticky Wax; Kerr Inc.)

$3 \mathrm{M} \mathrm{KCl}$

\section{EQUIPMENT}

Borosilicate glass capillaries (outer diameter, $2.0 \mathrm{~mm}$; inner diameter, $1.4 \mathrm{~mm}$; length, $85 \mathrm{~mm}$; Hilgenberg)

Carbon fibers (Thornel T650/42, 5- $\mu$ m diameter; Amoco Co.)

Patch-clamp rig on vibration-isolation table with inverted microscopeand micromanipulators, as described 16,17 , equipped with the components described in Table 1

Pipet and CFE fabrication equipment (Table 2)

Polyethylene(PE) tubing (inner diameter, $0.4 \mathrm{~mm}$; outer diameter, $0.8 \mathrm{~mm}$; SI M S Portex Ltd.)

$0.22-\mu \mathrm{m}$ syringe filters ( 3 - $\mathrm{mm}$ diameter)

Patch pipet filling needle (World Precision Instruments) 
Rig setup and calibration

\section{PROCEDURE}

1| Make connections on the patch-clamp rig according to Figure 1. Configure the data acquisition system to continuously record five traces for at least $30 \mathrm{~min}$ at a sampling rate $\geq 1 \mathrm{kHz}$. The five recorded traces are the lock-in amplifier outputs $\mathrm{CH} 1(\mathrm{Y} 1)$ and $\mathrm{CH}_{2}(\mathrm{Y} 2)$, the filtered patch-clamp amplifier current monitor $\mathrm{I}$, the filtered patch-clamp amplifier stimulus monitor $\mathrm{V}$, and the filtered amperometric current $A$.

A digital-to-analog $(D / A)$ output controls the holding potential and provides pulses for seal formation. A second $\mathrm{D} / \mathrm{A}$ or transistor-transistor logic ( $T \mathrm{TL}$ ) output provides a trigger signal to synchronize the oscilloscopes with the pulses. One digital output provides a TTL signal to switch the adder.

2| Mount the headstage of the amperometric amplifier on the micromanipulator to patch the cells and mount the headstage of the patch-clamp amplifier near the recording chamber.

Note that an $\mathrm{Ag} / \mathrm{AgC}$ ( silver-silver chloride) wire will be connected to the BNC input pin of the patch-clamp amplifier headstage and immersed into the bath (Step 16). Therefore, do not make any connections to the ground pin of the headstage of the patch-clamp amplifier, because the ground electrode will be connected to the BNC shield of the amperometric amplifier headstage.

3| Add image acquisition capability (optional). I mage acquisition capability can be used to store images of the pipet tip on the cell for later determination of the distance between the patch membrane and the tip of the CFE. An inexpensive USB or FireWire camera is sufficient. Alternatively, the distance can be measured during the experiment with an eyepiece micrometer in the microscope.

4| Set the lock-in amplifier SR830 as indicated in Table 3. Set EPC-7 STIM scaling to 0.1, which produces a $50-\mathrm{mV}$ r.m.s. pipet voltage from the $0.5 \mathrm{~V}$ sine wave provided by the lock-in amplifier.

Note that the scaling factors for the Axopatch 200B differ for the front panel- and back panel-switched inputs. If the front panel-switched input is used, then the scaling factor is 0.02 , and the lock-in output voltage must be set to $2.5 \mathrm{~V}$ to obtain a 50-mV r.m.s. pipet voltage signal. For the EPC-7, built-in filter 1 is set to $10 \mathrm{kHz}$ to avoid clipping because a $20-\mathrm{kHz}$ sine wave is used. The current monitor output is fed into the lock-in amplifier input via the $10 \mathrm{k} \Omega: 100 \mathrm{k} \Omega$ voltage divider because the output range of the EPC-7 is $\pm 10 \mathrm{~V}$, whereas the maximum input range of the lock-in amplifier is only $1 \mathrm{~V}$ r.m.s. Because the whole-cell mode of the Axopatch uses a smaller feedback resistor, lower gain may be used.

5| Find the approximate phase setting for the lock-in amplifier by slightly varying the C-slow compensation of the EPC-7. This may be done with open headstage. At correct phase setting, the lock-in amplifier output $Y 1$ is proportional to the membrane conductance and the output $\mathrm{Y} 2$ is proportional to the membrane capacitance ${ }^{1}$.

(i) Set EPC-7 to voltage-clamp mode, gain to $50 \mathrm{mV} /$ $\mathrm{pA}$; C-slow compensation to the 10-pF range, C-slow to $0.2 \mathrm{pF}$ (minimum), G-series to $0.2 \mu \mathrm{S}$, series resistance compensation to off; filter 1 to $10 \mathrm{kHz}$, filter 2 to $3 \mathrm{kHz}$; stimulus scaling to 0.1 , stimulus input filter $T_{R}$ switch to $2 \mu \mathrm{s}$.

The selected C-slow and G-series settings were found to give a time constant of $\sim 1.9 \mu$ s (ref. 3 ), which is close to typical patch time constants.

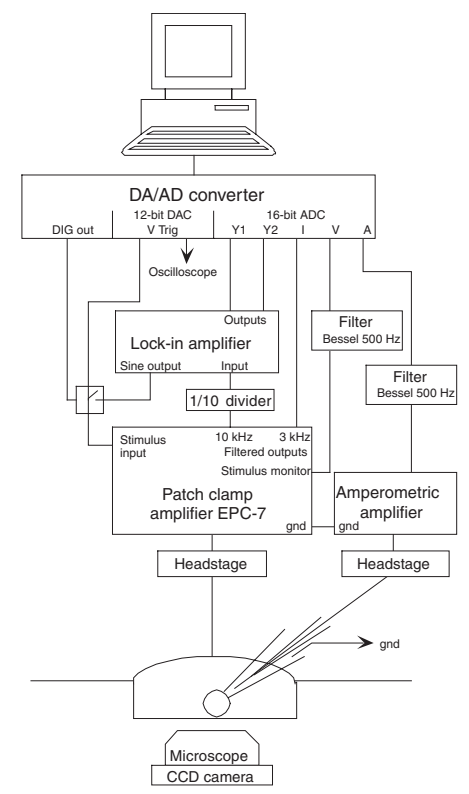

Figure 1 | Patch amperometry setup. Note that the electrode configuration is reversed: the bath electrode is connected to the input of the EPC-7 headstage while the ground electrode is inside the pipet. For low noise, connect a short $(<20$ $\mathrm{mm}$ ) chlorinated silver wire as bath electrode directly to the headstage input pin. Signal ground of the patch-clamp amplifier and of the amperometric amplifier are connected to the point of common ground that we choose to be on the vibration isolation table. Two oscilloscopes are omitted in this drawing and used to display four signals: (i) stimulus input of the patchclamp amplifier, (ii) current output filtered at $10 \mathrm{kHz}$, (iii) current output filtered at $3 \mathrm{kHz}$ and (iv) amperometric current filtered at $100 \mathrm{~Hz}$. 
(ii) Apply sine wave to stimulus input and adjust $\mathrm{C}$-fast and $\tau$-fast to null the current.

(iii) Slightly vary C-slow, ensuring that the EPC-7 is not saturated (not 'clipping') and change the phase setting of the lock-in amplifier. At the correct phase, changes in C-slow compensation appear only in the $\mathrm{CH} 2$ output (Y2, imaginary part of pipet current) with no change in $\mathrm{CH} 1$ output (Y1, real part). Note that an increase in C-slow compensation must produce a decrease in Y2 (capacitance decrease). This phase setting changes if any filters are changed or the gain is changed to the lowgain range. For EPC amplifiers always use $50-\mathrm{mV} / \mathrm{pA}$ gain for low-noise recording. The procedure is analogous for the Axopatch 200B in the 'whole-cell $\beta=1$ mode' choosing the corresponding settings for SERI ES RESTANCE ( $5 \mathrm{M} \Omega$ ) and WHOLE CELL CAP (minimum). A gain lower than $50 \mathrm{mV}$ / pA may be chosen because all gain settings use the same feedback resistor in the headstage.

6| Using the calibrated C-slow compensation, you may apply a defined change such as $200 \mathrm{fF}$. This, however, produces a large capacitive current that may saturate the amplifier (clipping). Thus, for calibration, the stimulus scaling must be switched to 0.01 (using EPC-7) giving a ten times smaller signal. This does not affect the phase setting. Now record the change of the $\mathrm{CH} 2$ output for the 200-fF C-slow change. Because the actual recordings from cells will be done with stimulus scaling set to 0.1, this $\mathrm{CH} 2$ output change corresponds to a 20 -fF change in a cell recording. In the PA_Acquire program, a calibration value is entered to convert the data into capacitance and conductance units.

For convenience we modified the patch-clamp amplifier by inserting a switchable $20-\Omega$ resistor in series with the C-slow potentiometer of the EPC-7. This capacitance dither applies capacitance compensation changes of $20 \mathrm{fF}$ for calibration. In the Axopatch 200B, the built-in 100-fF capacitance dither may be used, but make sure the gain is low enough to avoid saturation of the amplifier. It is possible to change the dithering circuit to provide a 10- or 20 -fF signal following guidelines available on request from Axon Instruments.

The pipet current (I) is filtered with the built-in 3-kHz filter 2 to suppress the $20 \mathrm{kHz}$ sine wave and is acquired by the analog-to-digital converter (ADC). An additional $500 \mathrm{~Hz}$ low-pass filter (optional) is recommended to filter the pipet current. The stimulus voltage ( V) is filtered at $500 \mathrm{~Hz}$ to suppress the $20 \mathrm{kHz}$ sine wave and acquired to monitor holding potential changes.

7| Set the voltage on the amperometric amplifier to $+700 \mathrm{mV}$ and gain to $10-40 \mathrm{mV} / \mathrm{pA}$, and acquire the amperometric current (A) filtered at $500 \mathrm{~Hz}$.

8| Prepare recording chambers by drilling a 10-mm hole in the center of the tissue culture dishes normally used in the setup and glue a 12 -mm coverslip underneath.

Preferably use Sylgard as glue because it is nontoxic and broken coverslips are easily replaced. Because the electrode configuration is reversed (the patch-clamp electrode in the bath), the stray capacitance of a bigger bath surface cannot be compensated. The smaller surface also reduces noise.

9| For the experiment, place a small coverslip with cells on the glass bottom of a dry chamber and fill with bath solution only into the inner well (typically $100 \mu \mathrm{l}$ ). Do not spill the bath solution onto the surrounding plastic because overflowing and evaporation of the solution gives rise to cycles of large stray capacitance changes, making recordings extremely difficult.

It is important to use an objective with long working distance to minimize stray capacitance. We use a Zeiss $20 \times 0.5$ Plan-Neofluar. An immersion objective would produce a very high stray capacitance that cannot be compensated.

10| Pull patch pipets with a tip diameter of $2-3 \mu \mathrm{m}$, a taper angle at the very tip of at least $20^{\circ}$ and a taper length of $\sim 5 \pm 0.5 \mathrm{~mm}$. An 85 - $\mathrm{mm}$ capillary will yield two pipets of $\sim 47 \mathrm{~mm}$. A large opening angle is necessary to bring the tip of the CFE close to the pipet tip.

Although puller settings need to be found experimentally, the following may serve as a guideline: the Sutter P-97 puller set up with a box filament of $3 \times 3 \times 3 \mathrm{~mm}$. Air pressure, 500; the air before pull and the air after pull, $5 \mathrm{~s}$ each. The ramp test result for the capillaries was 655 . A single line program looped four times and had the parameters: heat, 615; pull, 0; velocity, 50; and time, 200. For larger taper angles the velocity might be reduced slightly without increasing the tip diameter substantially. Follow the P-97 manual to modify settings depending on ramp test result. 
Table 1 | Components of the patch clamp rig

Comments
Patch-clamp amplifier: We obtained the best results with
the EPC-7. Although the EPC- 8 has a built-in 20 - $\mathrm{kHz}$ filter
that could be advantageous, amplifier clipping is a problem
at this setting. The EPC- 8 has only one filter switch, but has
two internal filters. Filter 1 is particularly important at high
gain to avoid clipping. Because filter 1 becomes active only
at filter settings $\leq 5 \mathrm{kHz}$, clipping occurs more frequently in
the high-gain range when a filter setting of $10 \mathrm{kHz}$ is used.
The Axopatch $200 \mathrm{~B}$ can be used with 'whole-cell $\beta=1$ ' mode.
Thus far, we have been unable to obtain patch amperometry
recordings in the capacitive feedback 'patch' mode because
there is excessive noise in this mode. Other amplifiers were
not tested. In the EPC-10 amplifier (HEKA Electronics) filter
1 can be set independently as in the EPC- 7 thus this amplifier
may be more suitable.

\section{Amperometric amplifier with grounded headstage BNC} shield: The EPC-10 dual amplifier may be very practical because it would also replace the patch-clamp amplifier. When ordering a VA10 or EPC-10, specify a special version with a head stage in which the head stage BNC shield is grounded. Patch amperometry measurements must use a modified VA10 or EPC-10 headstage because the BNC shield in the standard configuration is connected to 'Vref.' This standard headstage cannot be used because the reference electrode in the holder is connected to the BNC shield. Modified headstages in which the BNC shield is connected to ' $g$ nd' are available for the VA10 and EPC-10 but must be specially ordered. The schematics of a simpler version of an amperometric amplifier can be obtained from the Alvarez de Toledo laboratory.

Patch amperometry pipet holder: A schematic for a holder that fits the Axopatch 200B is available elsewhere ${ }^{18}$.

Low-pass filters: Because the maximal patch-clamp amplifier output is $\sim 10 \mathrm{~V}$ but the maximal Lock-in amplifier signal input is $1 \mathrm{~V} \mathrm{r.m.s.,} \mathrm{a} 10 \mathrm{k} \Omega: 100 \mathrm{k} \Omega$ 1:10 voltage divider must be inserted at the SR 830 signal input ${ }^{3}$.

\section{Computer-controlled analog signal adder: This optional} device adds the sine wave from the lock-in to the holding potential and pulses as generated by the D/ A converter. A TTL signal switches addition of the sine wave on and off. A circuit diagram is provided in Supplementary Figure 1. The Axopatch 200B has two command signal inputs that are added internally, one being selected or deselected by a front panel switch. With this amplifier the signal adder is thus not necessary but the sine wave must then be switched on and off manually.

\section{Data acquisition system with at least five channels} and a sampling rate $>\mathbf{1 ~ k H z : ~ A n ~ I G O R ~ e x p e r i m e n t ~ f o r ~}$ data acquisition 'PA_Acquire' is available (Supplementary Software online).

\begin{tabular}{ll} 
Component & Source \\
\hline EPC-7 & HEKA Electronics \\
Axopatch 200B & Molecular Devices
\end{tabular}

VA10, modified version

EPC-10 double, modified version
NPI Electronics

HEKA Electronics
Available from the Lindau and Alvarez de Toledo research laboratories

Stanford Research Systems example, 900 series 8 pole Lock-in amplifier SR 830 RG58C/U type coaxial Newark Electronics cables with $50-\Omega$ impedance 
Table 2 | Pipet and CFE fabrication equipment

\begin{tabular}{|c|c|c|}
\hline Equipment & Source & Comments \\
\hline Pipet puller (for example, P-97) & $\begin{array}{l}\text { Sutter } \\
\text { Instruments }\end{array}$ & \\
\hline Microforge for pipet conditioning & $\begin{array}{l}\text { ALA Scientific } \\
\text { Instruments } \\
\text { Inc. }\end{array}$ & For example, CPM-2 mounted on a suitable microscope \\
\hline Hot plate & & To melt sticky wax for pipet coating \\
\hline Recording chamber & & See Step 8 \\
\hline Watchmakers forceps & TechniTools & $\begin{array}{l}\text { For example, \#5; with tips protected by } 0.5 \mathrm{~cm} \text { of PE } \\
\text { tubing }\end{array}$ \\
\hline CFE-pulling device & $\begin{array}{l}\text { To be } \\
\text { assembled } \\
\text { see Step } 13\end{array}$ & $\begin{array}{l}\text { Components: Stereo microscope, power supply ( } 12 \mathrm{~V} \text {, } \\
>3 \mathrm{~A} \text { ), foot switch, } 0.4 \mathrm{~mm} \text { diameter } \sim 5-\mathrm{cm} \text {-long- } \\
\text { platinum-iridium ( } \mathrm{Pt} / \mathrm{Ir} \text { ) wire }\end{array}$ \\
\hline
\end{tabular}

11 Inspect each pipet after pulling for proper geometry and dip the pipet tip in melted wax to reduce stray capacitance. I mmediately before use, fire-polish the pipets with a microforge: approach a heated wire with the pipet tip such that the wax flows out and the tip narrows slightly.

It is essential to coat pipets with wax or with Sylgard as previously described ${ }^{16}$. The coating must cover a sufficient part of the tip such that it protrudes well beyond the bath surface to minimize stray capacitance and bath-pipet capacitance fluctuations. Once the pipet is assembled into the electrode holder (Steps 18-20) with the tip immersed into the bath, the resistance should be 1-3 $\mathrm{M} \Omega$.

12 Cut an $\sim 12 \mathrm{~cm}$-long piece of PE tubing, and immerse one end in a beaker with ethanol, so that the entire tube fills by capillary action. Using watchmaker forceps, with tips protected by PE tubing, insert a carbon fiber of at least $5 \mathrm{~cm}$ in length into the PE tube. Wick away ethanol by tapping the tube on filter paper. Evaporate the remaining ethanol at $50{ }^{\circ} \mathrm{C}$ for $30 \mathrm{~min}$.

This procedure was modified from the preparation described elsewhere ${ }^{7}$.

13| Set up the CFE-pulling device.

(i) Bend a platinum-iridium (Pt/Ir) wire of $\sim 5-\mathrm{cm}$ length and a diameter of $0.4 \mathrm{~mm}$ into a loop of $\sim 6$ - mm diameter and connect the wire via a foot switch to a variable power supply $(12 \mathrm{~V},>3 \mathrm{~A})$.

(ii) Place the loop under the dissection microscope and adjust the heat of the wire loop by holding a piece of PE tubing in the center of the wire loop (wire resistance of $\sim 1 \Omega$, current $\sim 2 \mathrm{~A}$ ). After the heat is switched on, it should soften within 10-15 s and melt within 20-25 s.

Table 3 | SR830 lock-in amplifier settings

\begin{tabular}{|c|c|}
\hline Variable & Setting \\
\hline Time constant & $1 \mathrm{~ms}, 24 \mathrm{~dB}$ \\
\hline Signal input & Input, $A$; couple, AC; ground, FLOAT \\
\hline Sensitivity & $1 \mathrm{~V}$ \\
\hline Reserve & LOW NOISE \\
\hline Filters & LI NE $\& 2 \times$ LI NE \\
\hline Channel 1 & Output X, OFFSET Off, RATI O Off, Expand Off \\
\hline Channel 2 & Output Y, OFFSET Off, RATIO Off, Expand Off \\
\hline Interface & RS232, 8, 9600, none \\
\hline Reference & Phase +120a , Freq 20 kHz, Ampl. $0.5 \mathrm{~V}^{\mathrm{b}}$, Harm \# 1 Trig SI NE, Source INTERNAL \\
\hline
\end{tabular}


14| To pull CFEs:

(i) Hold a carbon-fiber-filled tube with both hands in the middle of the heated wire loop, melt the PE and pull very gently. Pull on one side $(\sim 1 \mathrm{~cm})$ and push on the other side about half as much. This creates a tapered electrode that will fit nicely in the patch pipet (Fig. 2).

\section{$\triangle$ CRITICAL STEP}

(ii) Allow the PE to melt around carbon fiber as a thin layer. Do not let both parts separate yet.

\section{$\triangle$ CRITICAL STEP}

(iii) With a fresh scalpel blade, separate both parts on a clean glass surface.

(iv) Approach the heated wire with the carbon fiber tip such that the thin layer of PE melts back and forms a small bead.

(v) In a quick movement, switch off the heat applied to the wire, touch the wire with the small PE bead and pull the CFE away from the wire quickly. This forms an insulating layer of PE of $\sim 0.1-\mu \mathrm{m}$ thickness around the carbon fiber. Instructions on how to set up a programmable CFE puller are available in Supplementary Note 1 online.

15 Before use, recut the tip of the CFE to expose a freshly cut surface. Backfill the CFE with $3 \mathrm{M} \mathrm{KCl}$ with a syringe via a pipet-filling needle. On retracting the needle from the CFE, apply slight suction to the syringe so that drops in the end of the PE tubing will be sucked away. Dry the inside end of the CFE with a piece of tissue, as drops at the end of the PE tube of the CFE may lead to short circuits once the CFE is mounted to the holder. Cut the CFE to fit into the respective electrode holder. Typically the total length is $\sim 90 \mathrm{~mm}$.

Electrode and holder assembly
16| Install two silver wires in the electrode holder:

(i) For the manual holder (Fig. 3a), connect one wire to the center pin of the BNC plug on the holder; this wire will go inside the CFE tubing. Fix a $5 \mathrm{~mm}$ - long piece of PE tubing with an inner diameter of $0.8 \mathrm{~mm}$ to the inside of the BNC plug. This helps to hold the CFE in place and reduces the risk of short circuits.

(ii) In the motorized version (Fig. 3b), the wire is cannulated through the inner tube of the Nanomotor, which can move the CFE forward and backward using an external controller. Attach a 5-mm silicone tubing to the moving inner tube of the Nanomotor to mechanically connect the CFE to it.

(iii) For each holder, connect the other wire to the housing and thus to ground- the wire will be outside the CFE but inside the patch pipet.

(iv) Cut the 'ground' wire (connected to the electrode holder housing) to be shorter than the other (the 'CFE' wire connected to the central pin), to allow the two wires to be distinguished when the holder is assembled. Thoroughly chlorinate both wires.

17| Complete assembly of the electrode holder by mounting the front part that will hold the pipet. For the manual holder turn the fine-threaded cap until about one-half to two-thirds of the thread is exposed. The housing and the cap should not tilt against each other when suction is applied.

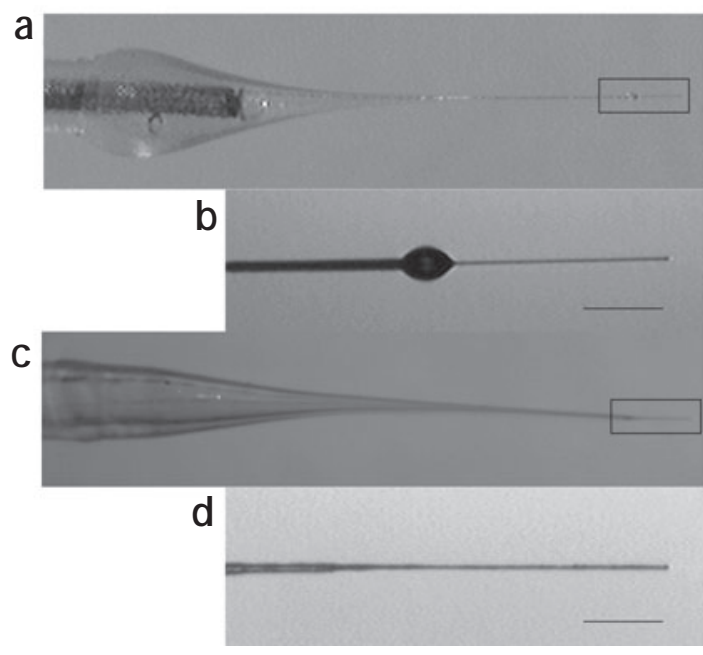

Figure 2 | Carbon fiber electrodes. (a) Overview of a conventionally fabricated CFE. Because of symmetrical pulling, there is a large PE bead on the left. Inside the large bead on the left, the silver wire $(0.25-\mathrm{mm}$ diameter) can be seen. (b) Magnification of the box in a with the small bead. The right part of the carbon fiber seems uncoated, but is insulated with a PE layer of about $0.1 \mu \mathrm{m}$. (c) The CFE prepared by asymmetrical pulling has almost no big bead. (d) Magnification of the box in c. The tip of the CFE lacks the small bead. The material of which the small bead consisted was melted and distributed over the thin part of the fiber so that it is covered with a slightly thicker layer of PE than the CFE in $\mathbf{b}$. Scale bars in $\mathbf{b}$ and $\mathbf{d}, 100 \mu \mathrm{m}$. Such a CFE is ideal to be inserted into the patch pipet and its tip will easily be placed at the pipet tip. 
18| Slide the $3 \mathrm{M} \mathrm{KCl}$-filled CFE (from Step 15) all the way onto the longer wire. For the motorized holder, use the end pushed into a small tubing that sits on the inner cylinder of the Nanomotor and connects the CFE to the motor.

19| After mounting the CFE into the holder, carefully insert the CFE tip into a pipet

filled with pipet solution, preferably under a dissection microscope or magnifying glass. Take care that the CFE does not bend backward, break or poke through the pipet tip. Adjust the length of the CFE, if necessary.

20| Turn the screw cap of the manual holder to bring the tip of the CFE close to the tip of the pipet (within 50-100 $\mu \mathrm{m}$ ). For the motorized holder, adjust the CFE to the same position using the Nanomotor. Be careful not to push through the pipet tip with the fiber, because this will not only destroy the pipet, but also scrape off the

thin PE insulation on the CFE tip and might lead to higher offset in the amperometric recording. Push the pipet all the way to the stop in the holder, secure with the nut and mount the electrode holder on the headstage in the patch-clamp rig.

\section{$\triangle$ CRITICAL STEP}

21| When the CFE is mounted on the amperometric headstage, assess the CFE quality.

(i) Apply the +700 -mV potential and observe the current: the amperometric current should decrease slowly and reach a level that represents a CFE resistance of 35 to $140 \mathrm{G} \Omega$ within 1 to $2 \mathrm{~min}$ ( 20 to $5 \mathrm{pA}$ at $+700-\mathrm{mV}$ stimulation voltage). The noise of the amperometric current should be $<1$ pA r.m.s.

(ii) (optional) Test CFE sensitivity: I mmerse the assembly into the bath solution and put slight positive pressure to the pipette. Add $10 \mu \mathrm{l}$ of $1 \mathrm{M}$ dopamine to $100 \mu \mathrm{l}$ of bath solution. Upon release of the positive pressure an amperometric signal will quickly evolve. The closer the CFE is to the pipette tip, the faster the signal will rise. Wash bath chamber thoroughly after this test.

\section{$\Rightarrow$ TROUBLESHOOTING}

22 Wash a small coverslip, or a piece of one, with adherent cells in bath solution and place it in the recording chamber, add about $100 \mu \mathrm{l}$ of bath solution (remember not to spill solution on the plastic surrounding the inner well), immerse the electrode of the patch-clamp headstage in the bath and lower the pipet tip into the solution.

23 Switch on the test pulses and measure the pipet resistance. Focus on the pipet tip in the inverted microscope. Adjust the CFE tip as described below for the manual (i) or for the motorized (ii) holder. At the final CFE position, acquire an image or measure the distance with the eyepiece micrometer or from the video screen.

(i) With the manual holder, carefully turn the cap of the holder to approach the pipet tip with the CFE tip until they are 5-10 $\mu \mathrm{m}$ apart (one to two times the CFE diameter).

Be careful not to poke the CFE through the pipet tip as this might scratch off the thin insulation layer on the CFE. A longer distance will broaden and flatten amperometric signals because of diffusion. It is difficult to record amperometric signals at distances $>30 \mu \mathrm{m}$. At too-short a distance, the CFE might interfere with seal formation as the patch of the cell is sucked into the pipet during sealing.

\section{CFE testing}

\section{Recording preparation}

\section{Adjusting the CFE to the pipet tip}


Patching and recording exocytotic events (ii) With the motorized holder, the seal is formed while the CFE is still at some distance from the tip - thus facilitating seal formation. Once the seal is formed, move the CFE close to the patch using the Nanomotor.

With experience, most users find the motorized holder much more convenient than the manual holder, because the motorized version substantially increases the number of recordings that can be made per day.

\section{TROUBLESHOOTING}

24| Use low patch-clamp amplifier gain and give test pulses. Put slight positive pressure on the pipet and approach a cell using the micromanipulators. Upon touching a cell, the pipet resistance will slightly increase. Release positive pressure and apply suction until pipet resistance reaches several gigaohms. When the motorized holder is used, adjust the CFE tip to be close to the patch. To reduce noise it may help to turn off the controller and disconnect the cable from the controller. For general patch-clamp instructions follow the excellent description available elsewhere ${ }^{17}$.

\section{$\Rightarrow$ TROUBLESHOOTING}

25 Increase the gain of the EPC-7 to $50 \mathrm{mV} / \mathrm{pA}$ and set the stimulus scaling to 0.1 . Adjust the C-fast and $\tau$-fast compensation.

26| Switch off the test pulses, switch on the sine wave and start data acquisition.

(i) Readjust C-fast and $\tau$-fast to compensate remaining capacitive currents until the sine wave current has minimal amplitude on the oscilloscope.

(ii) Apply some capacitance compensation changes for calibration (if necessary at reduced stimulus scaling; see Step 6). Try to complete these adjustments within a few seconds after achieving a gigaseal, because the cells release most releasable vesicles at the beginning of the recording induced by the mechanical stimulus of patching.

(iii) Observe the sine wave amplitude of the patch-clamp current monitor during the experiment and ensure that it does not exceed $\pm 10 \mathrm{~V}$, even if the EPC-7 clipping indicator might not be on. Readjust the $\mathrm{C}$-fast and $\tau$-fast compensation to decrease the current amplitude.

An exocytotic event can be identified by the synchronous appearance of an amperometric spike, a transient increase in $\mathrm{Y} 1$ indicating a narrow fusion pore and a step in the $\mathrm{Y} 2$ trace (proportional to the membrane area increment).

\section{TROUBLESHOOTING}

27| Monitor the end of the experiment. The end is marked either by losing the seal or by the cell going to the whole-cell configuration. In the latter case the cytosolic catecholamines diffuse out of the cell and cause a rather slow but fairly big wave in the amperometric current ${ }^{18}$.

\section{TROUBLESHOOTING TABLE}

PROBLEM

Step $\mathbf{2 1}$ There is no current in amperometric amplifier.

\section{SOLUTION}

The amplifier is not connected properly; recheck wiring and verify proper connections.

CFE is broken or is not immersed in $\mathrm{KCl}$ at tip. Check CFE under the microscope and, if broken, prepare new CFE.

Step 23 Amperometric amplifier is saturated. There is a short circuit due to wet end of CFE. Dry the inside of the holder and inside of CFE tubing.

Amperometric amplifier is saturated (clipping, signal overload) or there is high offset current.

Step 24 Amperometric spikes are observed without cell There is a defective chlorination layer on silver wires. contact.

Pipet moves considerably $(5-10 \mu \mathrm{m})$ when suction is applied.
There is bad insulation of CFE tip. Recut tip or prepare new CFE more carefully; do not remove insulation from carbon fiber tip. Rechlorinate silver wires thoroughly. housing and cap of electrode holder. 


\section{TROUBLESHOOTI NG TABLE ( cont.)}

PROBLEM

Step 26 Capacitance steps are observed without an amperometric spike.

Amperometric spikes are observed without a capacitance change (Y2 trace).

Capacitance steps are not confined to the $Y 2$ trace but have a projection in $\mathrm{Y} 1$.

\section{CRITICAL STEPS} fibers first until the described shape and taper is achieved. but it will bulge out and become a wider tube.

\section{SOLUTION}

The CFE is too far from the patch, the CFE does not work or the vesicles were empty ${ }^{12}$.

Check CFE position (Step 23), and check CFE sensitivity (Step 21 (ii)).

There is transient breakage of the cell during sealing resulting in freely floating vesicles outside the cell or clipping of the patch-clamp amplifier. Make sure C-fast and $\tau$-fast are properly adjusted (Step 26 (i)).

The patch clamp amplifier is clipping or the phase of the patch is different from the preset phase. The latter problem may be corrected offline, but clipping cannot be corrected for. To test the phase setting, apply gentle suction pulses, which produce capacitance changes; these changes will likely have the same projection in $Y 1$ as the capacitance steps from exocytotic events.

Step 14 (i) Manual pulling of PE tubing requires some practice. Pull just the PE tubing without carbon

Step 14 (ii) When pulling with a carbon fiber, make sure that PE melts around the fiber and does not just form a very thin tube. The latter will not form a nice retracting bead in the second melting stage,

Step 20 Once the holder is assembled with CFE and pipet, the solutions can indeed flow along the wires and follow gravity. Solution flowing backward might cause short circuits, so try always to keep the pipet pointing downward.

\section{COMMENTS}

The method will be enormously powerful for understanding themechanisms that regulate quantal sizein catecholaminergic neurons and endocrine cells sas well as the molecular properties of exocytotic fusion pores. It may be applied to all cell typesthat release oxidizable transmitters such as norepinephrine, dopamineor serotonin. In addition, any cell typemay beloaded with dopamineor serotonin as a false transmitter. During the initial opening of the fusion pore, flux of catecholamines and fusion pore conductanceare measured simultaneously. A detailed description of fusion poreanalysis for cell-attached capacitancemeasurements and consideration for choosing the optimal sine wave frequency depending on vesiclesize to be studied has been provided elsewhere ${ }^{3}$. Combining patch amperometry with fusion pore analysis has facilitated the analysis of the relation between catecholamineflux during the amperometric foot signal and fusion pore conductance ${ }^{10}$. Specific mutations may be introduced into protein sequences believed to beinvolved in fusion pore formation to differentially affect fusion pore conductance and catecholaminepermeation. Thus, the analysis of the structure and function of the fusion pore may beaided

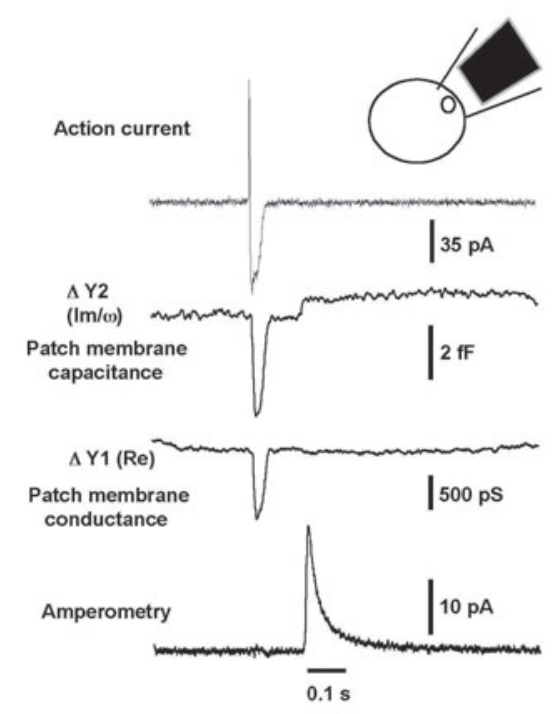

Figure 4 | Patch amperometry recording from a rat chromaffin cell. Catecholamine release (bottom trace) from a single granule fusion event (capacitance step in $\Delta \mathrm{Y} 2$ trace) after the firing of a spontaneous action potential as indicated by the action current (top trace). The top trace shows the patch current after low-pass filtering to remove the $20-\mathrm{kHz}$ sine wave. Such action currents recorded in a cellattached configuration are a mixture of capacitive and ionic currents through the patch membrane that are associated with action potentials ${ }^{25}$. The rapidly changing action current produces transient artifacts in $\Delta Y 1$ and $\Delta Y 2$ traces. 
by patch amperometry recordings of fusion events, thereby testing the role of specific protein domains in the exocytotic fusion pore.

\section{EXAMPLE OF APPLICATION}

Event analysis addresses quantal analysis, vesicular concentrations, considerations for patch capacitance measurements and fusion pore analysi $s^{2,3,10-14}$, as well as amperometric spike analysi $5^{6,19-24}$ and the relationship between cell electrical activity and fusion. An example of thetype of data obtained with the method is shown in Figure4. We providean IGOR experiment to view the recordings and to perform some analysis (see Supplementary Software).

\section{ACKNOWLEDGMENTS}

We thank L. Kwan and J . Lenz for the cell preparations and excellent technical assistance; M. Montesinos, R. Borges, R. Staal and D. Sulzer for critical reading of the manuscript and for contributing information on using the Axopatch 200B for patch amperometry. This work was supported by grants from the Deutsche Forschungsgemeinschaft, the National Institutes of Health (R01 NS38200) and the Nanobiotechnology Center (a Science and Technology Center (STC) program of the National Science Foundation, Agreement No. ECS-9876771) to M.L., and a grant from the Ministerio de Educación y Cultura, Spain to G.A.d.T.

\section{SOURCE}

This protocol was directly provided by the authors listed on the title page. Further details can be found in: Dernick, G. Simultaneous Detection of Fusion and Secretion by Patch Amperometry of Exocytosis of Small Vesicles (Berlin, Germany, 2000); available online (http:// www. dissertation.de/). For further information on microscopy, micromanipulators and CCD cameras, see Goldberg, R. \& Spector, D. Live Cell Imaging: A Laboratory Manual (Cold Spring Harbor Laboratory Press, Cold Spring Harbor, New York, USA, 2004).

1. Neher, E. \& Marty, A. Discrete changes of cell membrane capacitance observed under conditions of enhanced secretion in bovine adrenal chromaffin cells. Proc. Natl. Acad. Sci. USA 79, 6712-6716 (1982)

2. Lollike, K., Borregaard, N. \& Lindau, M. The exocytotic fusion pore of small granules has a conductance similar to an ion channel. J. Cell Biol. 129, 99-104 (1995)

3. Debus, K. \& Lindau, M. Resolution of patch capacitance recordings and of fusion pore conductances in small vesicles. Biophys. J. 78, 2983-2997 (2000).

4. Klyachko, V.A. \& J ackson, M.B. Capacitance steps and fusion pores of small and large-dense-core vesicles in nerve terminals. Nature 418, 89-92 (2002).

5. Wightman, R.M. et al. Temporally resolved catecholamine spikes correspond to single vesicle release from individual chromaffin cells. Proc. Natl. Acad. Sci. USA 88, 10754-10758 (1991).

6. Chow, R.H., von Rüden, L. \& Neher, E. Delay in vesicle fusion revealed by electrochemical monitoring of single secretory events in adrenal chromaffin cells. Nature 356, 60-63 (1992).

7. Chow, R.H. \& von Rüden, L. Electrochemical detection of secretion from single cells in Single Channel Recording, 2nd edn. (eds. Sakmann, B. \& Neher, E.) 245-275 (Plenum Press, New York, 1995).

8. Bruns, D. \& Jahn, R. Real-time measurement of transmitter release from single synaptic vesicles. Nature $\mathbf{3 7 7}, 62-65$ (1995).

9. Pothos, E.N., Davila, V. \& Sulzer, D. Presynaptic recording of quanta from midbrain dopamine neurons and modulation of the quantal size. J. Neurosci. 18, 4106-4118 (1998).

10. Albillos, A. et al. The exocytotic event in chromaffin cells revealed by patch amperometry. Nature $\mathbf{3 8 9}, 509-512$ (1997).

11. Alés, E. et al. High calcium concentrations shift the mode of exocytosis to the kiss-and-run mechanism. Nat. Cell Biol. 1, 40-44 (1999)

12. Tabares, L., Alés, E., Lindau, M. \& Alvarez De Toledo, G. Exocytosis of catecholamine-containing and catecholaminefree granules in chromaffin cells. J. Biol. Chem. 276, 3997439979 (2001)

13. Dernick, G., Alvarez De Toledo, G. \& Lindau, M. Exocytosis of single chromaffin granules in cell-free inside-out membrane patches. Nat. Cell Biol. 5, 358-362 (2003).

14. Gong, L.W., Alvarez De Toledo, G. \& Lindau, M. Secretory vesicles membrane area is regulated in tandem with quantal size in chromaffin cells. J. Neurosci. 23, 7917-7921 (2003).

15. Parsons, T.D., Coorssen, J.R., Horstmann, H. \& Almers, W. Docked granules, the exocytic burst, and the need for ATP hydrolysis in endocrine cells. Neuron 15, 1085-1096 (1995).

16. Hamill, O.P., Marty, A., Neher, E., Sakmann, B. \& Sigworth, F.J I Improved patch-clamp technique for high-resolution current recording from cells and cell-free membrane patches. Pflügers Arch. Eur. J. Physiol. 391, 85-100 (1981).

17. Penner, R. A practical guide to patch clamping in Single Channel Recording, 2nd ed. (eds. Sakmann, B. \& Neher, E.) 3-30 (Plenum Press, New York, 1995).

18. Mosharov, E.V., Gong, L.W., Khanna, B., Sulzer, D. \& Lindau, M. Intracellular patch electrochemistry: regulation of cytosolic catecholamines in chromaffin cells. J. Neurosci. 23 5835-5845 (2003).

19. Schroeder, T. . et al. Analysis of diffusional broadening of vesicular packets of catecholamines released from biological cells during exocytosis. Anal. Chem. 64, 3077-3083 (1992).

20. Jankowski, J.A., Schroeder, T.J., Ciolkowski, E.L. \& Wightman, R.M. Temporal characteristics of quantal secretion of catecholamines from adrenal medullary cells. J. Biol. Chem. 268, 14694-14700 (1993).

21. J ankowski, J.A., Finnegan, J.M. \& Wightman, R.M. Extracellular ionic composition alters kinetics of vesicular release of catecholamines and quantal size during exocytosis at adrenal medullary cells. J. Neurochem. 63, 1739-1747 (1994).

22. Wightman, R.M., Schroeder, T.J ., Finnegan, J.M., Ciolkowski, E.L. \& Pihel, K. Time course of release of catecholamines from individual vesicles during exocytosis at adrenal medullary cells. Biophys. J. 68, 383-390 (1995)

23. Schroeder, T.J. et al. Temporally resolved, independent stages of individual exocytotic secretion events. Biophys. J. 70, 1061-1068 (1996).

24. Segura, F., Brioso, M.A., Gomez, J.F., Machado, J.D. \& Borges, R. Automatic analysis for amperometrical recordings of exocytosis. J. Neurosci. Methods 103, 151-156 (2000).

25. Mazzanti, M. \& DeFelice, L.J . Na channel kinetics during the spontaneous heart beat in embryonic chick ventricle cells. Biophys. J. 52, 95-100 (1987). 